Aceitamos de bom grado e nímia satisfação, a incumbência de apresentar o Dr. Guilherme dos Reis Pereira Janson, pois já de há muito o conhecemos e o admiramos por sua inteligência, talento e dedicação às lides da Ortodontia.

Já na graduação demonstrava as qualidades imprescindiveis para um futuro profissional, detalhista e perfeccionista. Por isso, o convidamos para realizar o mestrado e o doutorado, distingüindo-se posteriormente como professor e pesquisador de renome.

Após o término do doutorado obtivemos uma bolsa da CAPES e o enviamos ao Prof. Woodside, em Toronto, no Canadá, onde também demonstrou talento e proficiência, finalizando seu pós-doutorado com distinguidos encômios. Indiscutivelmente, representa um dos professores bem reputados da FOB, imprescindivel como coordenador e professor do mestrado, formando profissionais que engrandecem a docência da Ortodontia junto às demais faculdades. É um mestre estrito, estudioso, que por sua competência e dedicação, angaria o respeito e a admiração de seus alunos.

De posse dos dados originais que nós, da equipe de professores do Departamento de Ortodontia da FOB-USP, haviamos angariado por 20 anos, o Dr. Guilherme, com paciência, dedicação e esforços incansáveis, editorou o "Atlas de Crescimento Craniofacial"; obra que constitui uma pesquisa inédita, de valor inaudito e irreprodutivel, de acordo com as normas éticas vigentes.

Com suas pesquisas, sua notoriedade ultrapassou fronteiras. Tanto que em novembro do ano passado, sua credibilidade foi reconhecida com um editorial do Dr. David Turpin, no American Journal Orthodontics and Dentofacial Orthopedics, sobre a correção de Classe II, subdivisão, referendando sete artigos, dentre os quais, quatro de sua autoria.

Como pesquisador crítico, compenetrado, rigoroso e ousado, o Dr. Guilherme conjuga sua ampla visão clínica com os assuntos mais polêmicos em suas investigações, fundamentando suas idéias e questionamentos com evidências cientificas. Indiscutivelmente é o pesquisador brasileiro que mais publica e publicou na literatura ortodôntica internacional, representando, portanto, a vocação do ortodontista brasileiro no exterior.

Dr. Décio Rodrigues Martins

\section{Guilherme Janson}

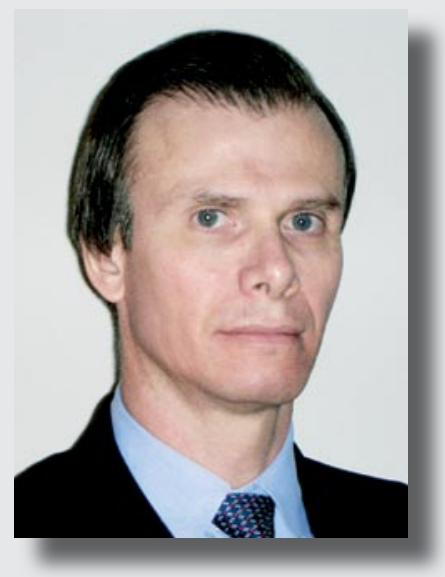

Professor Titular em Ortodontia da Faculdade de Odontologia de Bauru - Universidade de São Paulo (FOB-USP) - Departamento de Odontopediatria, Ortodontia e Saúde Coletiva;

Coordenador do Curso de Mestrado em Ortodontia da FOB-USP;

- Pós-doutorado na Faculdade de Odontologia da Universidade de Toronto - Canadá.

MRCDC - Member of the Royal College of Dentists of Canada.

- Membro da American Association of Orthodontists.

Membro da European Orthodontic Society.

- Diretor Científico da APEO.
1) No editorial da AJODO de novembro de 2005, o editor David Turpin, discorrendo sobre o tratamento dos casos de Classe II, subdivisão, referencia 7 artigos, dentre os quais, 4 são de sua autoria, atribuindo ao seu grupo de trabalho $o$ crédito pelas propostas concretas existentes na literatura para tratamento deste tipo de má oclusão. Resumidamente, como foi concebida e no que consiste esta sua visão do tratamento dos casos assimétricos, considerada bastante ousada para os moldes mais tradicionais? Maria Fernanda Martins-Ortiz Consolaro

A abordagem dos casos assimétricos foi baseada em nosso trabalho sobre as características da Classe II subdivisão e também em resultados de outras investigações sobre esse tipo de má oclusão, $1,36,51,62,76$. Estes trabalhos demonstraram que o principal fator que causa a Classe II subdivisão é o posicionamento distal dos primeiros molares inferiores do lado da Classe II. Secundariamente, essa má oclusão também pode ser causada pelo posicionamento mesial dos primeiros molares superiores do lado da Classe II, numa freqüência menor. Portanto, a abordagem consiste em se determinar qual o fator causador da Classe II subdivisão, por meio do exame clínico frontal do paciente, observando-se o posicionamento das linhas médias dentárias superior e inferior, em relação ao plano sagital mediano ${ }^{44,57}$. Se a linha média superior estiver coincidente e a inferior desviada, classifica-se a Classe II subdivisão como Tipo 1 (o molar inferior é que se encontra para distal no lado da Classe II) e se for o contrário, como Tipo 2 (o molar superior é que se encontra para mesial no lado da Classe II). Dentre os diversos protocolos de tratamento possíveis, no Tipo 1 (veja a resposta 
número 12 ao Dr. Osmar, com os diversos protocolos) a melhor opção é realizar o tratamento com extrações de três pré-molares (dois superiores e um inferior, do lado da Classe I), se o perfil do paciente permitir extrações. No Tipo 2, se o paciente não estiver em crescimento, a melhor opção é realizar o tratamento com extração de um pré-molar superior, do lado da Classe II. Assim, termina-se com um lado em Classe I de molar e com o outro em Classe II. Esses protocolos de extrações assimétricas não são novidade na Ortodontia ${ }^{15,16,89}$, entretanto, essa sistemática de abordagem é original. Alguns colegas mais tradicionais podem não admitir essas formas de correção por considerarem que os primeiros molares devem sempre terminar em relação de Classe I ao final do tratamento ${ }^{4,61,83}$. Entretanto, esse foi um conceito que dominou o pensamento ortodôntico durante algum tempo e que não encontrou suporte científico à medida que a especialidade se desenvolveu ${ }^{2,9}$.

2) Sua linha de pesquisa mais recente e 3 publicações internacionais exploram os protocolos de tratamento com extrações de 2 e 4 pré-molares, no tratamento da Classe II. Quais foram as observações clínicas que o levaram a desenvolver estas pesquisas? E quais seriam as principais vantagens da extração de 2 e não de 4 pré-molares, no tratamento da Classe II? Maria Fernanda Martins-Ortiz Consolaro

As observações clínicas foram que o tratamento com extrações de 2 pré-molares apresenta uma maior facilidade na mecânica ortodôntica, em comparação ao tratamento com 4 extrações, especialmente em uma Classe II completa. Aliás, essa dificuldade já foi bem demonstrada teoricamente por Andrews ${ }^{2}$. A maior dificuldade consiste em se relacionar os molares em Classe I. Para isso é necessária a colaboração do paciente em utilizar dispositivos extrabucais e/ou elásticos de Classe II para distalizar os molares superiores, redirecionar o crescimento maxilar e mesializar os molares inferiores. Como a colaboração requerida dos pacientes deve ser intensa, e como geralmente os pacientes não são muito colaboradores, isso faz com que a proporção de sucesso do tratamento com extrações de 4 pré-molares seja menor do que com extrações de 2 pré-molares superiores ${ }^{39}$. Portanto, a principal vantagem de se extrair 2 e não 4 pré-molares na Classe II completa é que a colaboração necessária do paciente será menor, o que implicará em uma maior proporção de sucesso oclusal, nos pacientes ${ }^{39}$. Outra vantagem, quando não houver apinhamento inferior, é que a quantidade de retração dos incisivos superiores e inferiores será menor, causando menor retrusão do tecido mole, quando este não pode sofrer grandes modificações.

3) Quais características clínicas o Sr. considera as principais e indicativas da necessidade de extrações dentárias? Maria Fernanda Martins-Ortiz Consolaro

As principais características clínicas que requerem extrações dentárias são o apinhamento acentuado e a protrusão desfavorável do tecido mole, associada ou não à ausência de selamento labial ${ }^{6,75}$. Quando o apinhamento é acentuado, não sendo possível obter-se o espaço necessário transversalmente ou anteroposteriormente, não há outra saída a não ser se recorrer às extrações dentárias ${ }^{82}$. A protrusão desfavorável do tecido mole se refere àquela em que o aspecto estético do perfil do paciente está prejudicado, causando principalmente uma ausência de selamento labial em repouso. Somente uma protrusão cefalométrica dos incisivos, sem reflexo negativo no tecido mole, não deve ser considerada uma motivação para extrações, de acordo com os conceitos ortodônticos contemporâneos ${ }^{73,78}$.

4) Tendo em vista sua publicação internacional mais recente ${ }^{48}$, quais são suas principais conclusões a respeito do planejamento e estabilidade da correção da mordida aberta anterior? Maria Fernanda Martins-Ortiz Consolaro

O que pudemos concluir sobre a estabilidade da correção da mordida aberta anterior na dentadura 
permanente é que se o tratamento é realizado sem extrações, a estabilidade clínica é de $61,9 \%$ e se realizado com extrações é de 74,2\%27,48,49. Estabilidade clínica refere-se à observação de um trespasse vertical, entre os incisivos superiores e inferiores de até $0 \mathrm{~mm}$, em longo prazo nos pacientes. Se o trespasse vertical se mostrasse negativo, nas telerradiografias, considerava-se que havia uma recidiva clínica. Portanto, os números acima se referem às porcentagens de pacientes que em longo prazo ainda demonstravam um trespasse de até $0 \mathrm{~mm}$ ou mais. Assim, consideramos que esses sejam os parâmetros mais objetivos que o clínico ortodontista pode ter em mente quando tratar seus pacientes de mordida aberta anterior, na dentadura permanente, quanto à esperada estabilidade dos resultados obtidos. Podese considerar que esses resultados não sejam muito animadores, porém, eles refletem o que realmente acontece. Como se sabe, os problemas de padrão de crescimento vertical são os mais difíceis de serem tratados e controlados e esses resultados demonstram mais uma vez a veracidade dessa afirmação. Portanto, o clínico deve ter especial atenção com esses pacientes para que realizem tratamentos fonoaudiológicos após o tratamento ortodôntico, se necessário, e instalar aparelhos de contenção que tendem a minimizar a recidiva, como placas com coberturas de acrílico oclusais na região posterior e grade palatina para ajudar a reduzir hábitos deletérios que possam estar associados. O estudo mais recente mencionado demonstrou que houve uma estabilidade estatisticamente maior nos casos tratados com extrações, em relação aos tratados sem extrações ${ }^{48}$. Entretanto, extrações não devem ser realizadas somente por esse motivo. São as outras características associadas à má oclusão que determinarão se o caso deve ou não ser tratado com extrações.

5) Considerando suas pesquisas e experiência junto a pesquisadores como Woodside, Metaxas, Voudouris e Angelopoulos, qual é o seu pensamento sobre os benefícios consistentes obtidos por aparelhos ortopédicos na fossa (mandibular ou glenóide) e côndilo mandibular? Maria Fernanda Martins-Ortiz Consolaro

As investigações realizadas na Faculdade de Odontologia da Universidade de Toronto demonstraram histologicamente que há remodelação da fossa glenóide (a literatura inglesa continua a denominá-la assim) e do côndilo mandibular como resposta à utilização de aparelhos ortopédicos funcionais $^{63,86,87,91}$. Essa remodelação consiste de um aumento de aproximadamente $1 \mathrm{~mm}$ a mais, em relação ao crescimento normal esperado. Quanto à estabilidade, a remodelação ocorrida na parede posterior da fossa glenóide foi perdida após oito semanas de remoção do aparelho, sendo mantida apenas na parede superior da fossa ${ }^{3}$. Quanto à remodelação ocorrida no côndilo, ela se apresenta maior, quando comparada a um grupo controle durante o tratamento e o tempo de contenção. Contudo, em longo prazo, não há diferença entre os tamanhos mandibulares do grupo tratado e do grupo controle ${ }^{14,85}$. Portanto, explica-se que os aparelhos funcionais estimulam as alterações que normalmente ocorreriam com o crescimento, de forma que ocorram mais rapidamente, durante o tratamento. Entretanto, posteriormente ao tratamento e ao período de contenção, as alterações no grupo experimental passam a ser mais lentas que no grupo controle, de forma que os tamanhos finais das mandíbulas nos dois grupos sejam semelhantes. Em outras palavras, os aparelhos funcionais não são capazes de proporcionar um comprimento mandibular maior do que o geneticamente determinado.

\section{6) Qual sua opinião sobre distalizadores in- trabucais e extração dentária? Francisco Anto- nio Bertoz \\ Os distalizadores intrabucais apóiam-se nos dentes anteriores e no palato para distalizar os molares, apresentando pequenas variações em seu desenho, dependendo da preferência de seu idea- lizador $8,13,25,30,34,52$. Uma vez distalizados os dentes posteriores, aparelhos extrabucais e/ou elásticos de Classe II devem ser utilizados para manter os den-}


tes posteriores em sua posição distalizada, enquanto os dentes anteriores são retraídos. Com algumas pequenas variações, basicamente essa é a abordagem utilizada com os distalizadores. Portanto, durante a primeira fase, de distalização dos dentes posteriores, há uma grande perda de ancoragem, causando uma protrusão dos dentes anteriores, que posteriormente terão que ser retraídos ${ }^{28}$. Isso pode causar insatisfação em alguns pacientes e em seus pais. Posteriormente, quando os dentes anteriores tiverem que ser retraídos, haverá necessidade de colaboração dos pacientes em utilizar aparelhos extrabucais e/ou elásticos de Classe II. Assim, os distalizadores na verdade não eliminam a utilização desses dispositivos e a conseqüente colaboração necessária, dos pacientes. Adicionalmente, a protrusão desfavorável dos dentes anteriores ocasionará um aumento do tempo de tratamento, porque os dentes anteriores terão que ter uma maior retração do que aquela inicialmente apresentada. Além disso, as requeridas modificações nos distalizadores intrabucais, para primeiramente distalizarem os segundos e depois os primeiros molares, demandam um grande tempo clínico do profissional. Em vista disso, eles são dispositivos pouco eficientes, especialmente quando comparados ao protocolo de tratamento da Classe II com extrações de dois pré-molares superiores ${ }^{72}$. Eficiência referese à maior quantidade de alteração oclusal obtida em um menor período de tempo ${ }^{5,38,65}$. Estudo recente demonstrou que a eficiência do Pendulum foi significantemente menor e o tempo de tratamento foi significantemente maior do que o protocolo de extrações de dois pré-molares superiores no tratamento da Classe $\mathrm{II}^{72}$. Poderia se argumentar que as duas extrações ocasionariam uma maior retrusão dos incisivos superiores, que pudesse modificar desfavoravelmente o perfil dos pacientes. Entretanto, estudo recente demonstrou que as quantidades de retração dos tecidos tegumentares são semelhantes quando a Classe II divisão 1 é tratada sem ou com extrações de dois pré-molares superiores, porque o trespasse horizontal a ser corrigido é o mesmo ${ }^{29}$. Além do mais, a distalização dos molares superiores diminui a probabilidade de espaço para a erupção dos terceiros molares superiores, requerendo que sejam extraídos posteriormente ${ }^{46,74}$. A extração nesses casos geralmente deve ser realizada por cirurgiões especializados e não simplesmente pelo clínico geral, que pode realizar as extrações dos prémolares ${ }^{55}$. Assim, o paciente terá sofrido extrações de dois dentes de toda forma.

\section{7) Ciente de sua experiência em Ortopedia com fundamentação científica como vê no sé- culo XXI o tratamento das más oclusões? Fran- cisco Antonio Bertoz}

A literatura é unânime em demonstrar que os aparelhos ortopédicos funcionais, no tratamento da má oclusão de Classe II, promovem principalmente alterações dentoalveolares e secundariamente alterações esqueléticas, nas respectivas proporções de 70 e 30\%7. Portanto, são capazes de corrigir esse tipo de má oclusão. O principal inconveniente da maioria dos aparelhos ortopédicos funcionais é que são removíveis e, por conseguinte, dependem da colaboração do paciente. Esse é um fator que contribui para diminuir sua eficiência ${ }^{5,11,12}$. A tendência atual é para que o ortodontista dependa cada vez menos da colaboração do paciente para atingir seus objetivos. Então, nesse sentido, os aparelhos ortopédicos funcionais que deverão prevalecer serão os fixos, como o aparelho de Herbst e suas variações; o aparelho de protração mandibular (APM), o Jasper Jumper e similares ${ }^{17-20,24,56,67,68,81,90}$.

\section{8) Na sua opinião quais as pesquisas reali- zadas nos últimos anos que contribuíram com a evolução da Ortodontia e como? Francisco} Antonio Bertoz

As pesquisas que mais contribuíram para a evolução da Ortodontia nos últimos anos foram aquelas que demonstraram que não são os valores cefalométricos baseados em padrões de normalidade que garantem o melhor resultado estético final do perfil dos pacientes e uma maior estabilidade para o alinhamento ântero-inferior, obtido com o 
tratamento ${ }^{26,58-60,80,92}$. Isso é muito importante, pois desde o advento da cefalometria o ortodontista passou muito tempo dando uma relevância demasiada a valores cefalométricos padrões e deixou de considerar a variação individual e a combinação dos distintos componentes da face do paciente. Por essa razão, realizaram-se demasiadamente tratamentos "procrustianos", que consistem em se tentar colocar todos os pacientes dentro de determinados padrões de normalidade a todo custo ${ }^{78,83}$. Os resultados dessa abordagem foram vários casos que tiveram uma deterioração de seu aspecto facial inicial, ao final do tratamento ortodôntico. Felizmente, o aspecto facial e a variação individual têm um papel mais relevante no diagnóstico e planejamento ortodôntico atualmente $^{78}$.

\section{9) Qual a sua opinião sobre o tratamento e} a finalização de todos os casos com o côndilo em relação cêntrica? Francisco Antonio Bertoz

Esse é o parâmetro ideal a ser observado para o diagnóstico e finalização dos casos ortodônticos, por ser considerada a posição mais fisiológica do côndilo dentro da fossa glenóide ${ }^{23}$. É importante que o diagnóstico seja realizado em relação cêntrica para que se avaliem corretamente as discrepâncias dentoalveolares e esqueléticas nos três planos do espaço ${ }^{45}$. Caso contrário, pode-se realizar um diagnóstico incorreto e conseqüentemente o plano de tratamento também será incorreto. Como exemplo, o diagnóstico incorreto de uma Classe III falsa e seu tratamento como Classe III verdadeira, que poderá ser bem mais complicado. $\mathrm{Na}$ Classe II, a finalização com o côndilo em relação cêntrica assume especial importância, senão muitos casos poderiam ser simplesmente terminados condicionando-se o paciente a ocluir em posição mais anterior para corrigir a discrepância ântero-posterior dessa má oclusão. Entretanto, na maioria das vezes esse condicionamento é temporário e quando perdido irá novamente demonstrar a quantidade de discrepância ântero-posterior que não foi devidamente corrigida ${ }^{77}$. Ou seja, o tratamento de uma má oclusão de Classe
II que não termine com o côndilo em relação cêntrica demonstra que a discrepância ântero-posterior original não foi completamente corrigida.

10) Qual sua opinião sobre o futuro da Ortodontia? Francisco Antonio Bertoz

O futuro da Ortodontia é brilhante, pois a tecnologia está possibilitando sua utilização em casos em que, anteriormente, pouco poderia ser realizado com os dispositivos e conhecimentos existentes. Braquetes autoligáveis, fios superelásticos e termoativados, e mais recentemente os mini-implantes modificaram bastante os paradigmas ortodônti$\cos ^{10,53,64,69,70,93}$. Todo esse desenvolvimento trouxe e continuará trazendo também modificações na forma em que o serviço é prestado aos pacientes. Para que o profissional desfrute de todos os benefícios que esse desenvolvimento trará, é necessário que se mantenha atualizado e com a mente aberta para as modificações que a especialidade exige, tanto na maneira como o tratamento é conduzido como no reflexo que isso provoca em relação à administração da prática clínica.

11) Quais os requisitos que o Sr. considera mais importantes para estabelecer os limites de um tratamento ortodôntico com compensações dentárias? Osmar A. Cuoghi

Primeiramente deve se conhecer qual a queixa principal do paciente. Se sua preocupação envolve deformidades esqueléticas, não haverá como corrigir esse problema somente ortodonticamente e o caso não poderá ser tratado com compensações dentárias, porque o paciente não ficará satisfeito. Se existe uma deformidade esquelética clínica, mas o paciente não a considera importante, então pode se realizar o tratamento com compensações dentárias, com a finalidade de corrigir as discrepâncias oclusais. Haverá uma melhora também do aspecto facial do paciente, muito embora, aquém do ideal que poderia ser obtido cirurgicamente. Entretanto, a preocupação principal do paciente foi solucionada e ele ficará satisfeito. Na Classe II, 
a extração de dois pré-molares superiores é uma maneira freqüente de se realizar uma compensação dentária dessa má oclusão, com resultados excelentes e bastante satisfatórios. Na Classe III, similar compensação pode ser realizada com extrações de dois pré-molares inferiores. $\mathrm{O}$ desenvolvimento de novos conceitos de tratamento tem permitido compensações acentuadas na má oclusão de Classe III. Exemplo de um novo conceito na Classe III é a prescrição de braquetes com torques linguais nos incisivos superiores e vestibulares nos incisivos inferiores para poderem se contrapor à ação de elásticos de Classe III $^{42}$. Especula-se que assim os dentes sejam submetidos a uma movimentação de corpo, o que favoreceria a remodelação óssea no sentido em que são movimentados, e conseqüentemente consigam possibilitar maiores alterações dentoalveolares ${ }^{31,88}$.

12) Quais são as condutas clínicas que o senhor considera mais viáveis para o tratamento da má oclusão de Classe II subdivisão? Osmar A. Cuoghi

Como já parcialmente explicado na primeira pergunta da Dra. Maria Fernanda (vide resposta), o primeiro passo para o tratamento de uma má oclusão de Classe II subdivisão é diagnosticar qual o fator causador do problema pela observação dos desvios das linhas médias dentárias superior e inferior, em relação ao plano sagital mediano. Posteriormente, a sistemática de abordagem está resumida abaixo:

Sistemática de abordagem de casos de Classe II subdivisão ${ }^{36,41,44,50,51,57}$ :

1) Classificar a má oclusão de Classe II subdivisão:

- Tipo 1: linha média superior coincidente e inferior desviada em relação ao plano sagital mediano;

- Tipo 2: linha média superior desviada e inferior coincidente com o plano sagital mediano;

- Tipo combinado: linha média superior para um lado e inferior para o outro.
2) Escolha do protocolo de tratamento para o problema:

- Protocolos de tratamento para o Tipo 1:

a) Paciente na dentadura mista

- Aparelhos funcionais removíveis ou fixos;

b) Paciente na dentadura mista com protrusão e ausência de selamento labial

- Extrações de 3 pré-molares (quando irromperem os permanentes);

c) Paciente na dentadura permanente precoce

- Elásticos de Classe II;

- Aparelhos funcionais fixos;

- Extrações de 3 pré-molares (se houver protrusão e ausência de selamento labial);

d) Paciente na dentadura permanente tardia

- Elásticos de Classe II;

- Extrações de 3 pré-molares (se houver protrusão e ausência de selamento labial);

- Protocolos de tratamento para o Tipo 2:

a) Paciente na dentadura mista ou permanente precoce

- Aparelho extrabucal assimétrico;

- Extração de 1 pré-molar;

b) Paciente na dentadura permanente tardia

- Extração de 1 pré-molar; - Protocolos para o Tipo combinado:

Levar em consideração as outras características da má oclusão para optar para tratar como Tipo 1 ou 2. Exemplos: se houver apinhamento moderado, protrusão e ausência de selamento labial, optar por tratar como Tipo 1, com extrações de 3 prés.

Se houver mínimo ou nenhum apinhamento e bom perfil facial, optar por tratar como Tipo 2, com extração de 1 pré ou com elásticos de Classe II.

13) Sabemos que os tratamentos ortopédicos funcionais têm proporcionado muitas dúvidas tanto com relação à sua resposta em curto como em longo prazo. Quais são as suas observações para esta modalidade de tratamento? Osmar A. Cuoghi

Os tratamentos ortopédicos funcionais são principalmente utilizados para a correção da má 
oclusão de Classe II e realmente são capazes de corrigir esse problema ${ }^{32}$. Entretanto, as respostas para o tratamento da Classe II são mais lentas que aquelas dos aparelhos fixos associados ou não a forças extrabucais ou elásticos de Classe II ${ }^{86}$. Portanto, para a correção de discrepâncias ânteroposteriores semelhantes, o tempo de tratamento com esses aparelhos é significantemente maior que o realizado com aparelhos extrabucais ${ }^{11,12}$. Os resultados de correção da má oclusão de Classe II, sejam quando tratada com aparelhos extrabucais e/ou elásticos de Classe II, ou aparelhos funcionais, serão estáveis se após a correção houver um tempo suficiente de contenção ativa ${ }^{40,54}$. Caso contrário, ocorrerá recidiva da discrepância ântero-posterior ${ }^{54}$. Com relação às alterações na fossa glenóide e no côndilo mandibular, a explicação se encontra na resposta da questão número 5 da Dra. Maria Fernanda.

14) $O$ que o Sr. considera mais viável para o tratamento da má oclusão de Classe III em pacientes que estão na fase de crescimento? Ortopedia Funcional dos Maxilares ou Ortopedia Mecânica? Osmar A. Cuoghi

O tratamento da má oclusão de Classe III durante a fase de crescimento é trabalhoso porque deve se lutar contra o crescimento desfavorável até o final deste. Para isso é necessário lançar mão dos dispositivos mais eficientes possíveis. Portanto, durante a dentadura mista, o protocolo recomendável é que após a expansão rápida maxilar se utilize a máscara facial para realizar a protração da maxila e/ou dos dentes superiores para se corrigir a mordida cruzada anterior. Após a sobrecorreção do descruzamento da mordida anterior e de um período de contenção com a própria máscara facial, utiliza-se o aparelho de Fränkel III durante o dia e a mentoneira à noite para redirecionar o crescimento desfavorável mandibular. Assim, o paciente deverá ser monitorado periodicamente para se garantir que não ocorra a recidiva do problema. Quando os dentes permanentes irrom- perem, a finalização com aparelhos fixos deve ser realizada mantendo-se a mentoneira à noite. Após o tratamento, a mentoneira à noite é mantida até o final do crescimento associada ou não ao aparelho de Fränkel III durante o dia. Se o paciente procurar o tratamento já na dentadura permanente, elásticos de Classe III corrigirão a mordida cruzada anterior. Após o descruzamento, utiliza-se a mentoneira à noite, como contenção. Finalizado o tratamento, seguir as demais orientações anterio$\mathrm{res}^{37}$. Dessa forma, percebe-se que para a correção do problema a utilização da Ortopedia Mecânica é mais eficiente e a Ortopedia Funcional é utilizada preferencialmente como um dispositivo de contenção ativa ${ }^{37,47}$.

15) Quando o senhor está trabalhando com dados numéricos obtidos por meio de uma pesquisa, qual o delineamento estatístico utilizado para a comparação dos dados? Além disso, na sua opinião o que define o "poder" de um teste estatístico? Osmar A. Cuoghi

Para o delineamento estatístico de comparação dos dados é necessário se conhecer o que se deseja comparar. De uma maneira simplista, se os dados forem paramétricos utiliza-se testes paramétricos e se forem categóricos, testes não-paramétricos. Mesmo com dados paramétricos, para que alguns testes sejam realizados, é necessário que os dados apresentem uma distribuição normal. Assim, antes dos testes serem realizados, os dados são submetidos a testes de normalidade com a finalidade de saber se os mesmos podem ser utilizados. Se não apresentarem distribuição normal, testes não paramétricos devem ser realizados. Para se investigar alterações resultantes do tratamento em um único grupo, de dados paramétricos, mais freqüentemente é utilizado o teste $t$ dependente. Se a comparação é entre grupos diferentes, utiliza-se o teste $t$ independente. Quando mais de dois grupos são comparados, utiliza-se a análise de variância. Estes são os testes mais freqüentemente utilizados. Entretanto, o delineamento dos testes a serem utilizados é variá- 
vel e depende muito do que se deseja investigar ${ }^{79}$. O poder de um teste estatístico é a probabilidade de que ele irá corretamente conduzir à rejeição de uma falsa hipótese nula. É a habilidade de um teste detectar se o efeito realmente existe. O poder estatístico é expresso por 1- $\beta$, onde $\beta$ (Beta) é o erro Tipo II, a probabilidade de não se rejeitar a hipótese nula quando ela é falsa ${ }^{21,33}$.

16) Diante da sua experiência clínica, respaldada pelas suas inúmeras pesquisas científicas, com evidência nos resultados encontrados, qual seria, em sua opinião, a melhor abordagem para o tratamento da má oclusão de Classe II em pacientes em crescimento e colaboradores? Nelson Mucha

Para se realizar o tratamento da má oclusão de Classe II, deve-se identificar a severidade da discrepância ântero-posterior. Quanto maior for essa discrepância, maior a dificuldade de correção da mesma, de tal forma que a maior dificuldade é se corrigir uma Classe II completa ${ }^{2,35}$. De uma forma geral, o tratamento da Classe II deve ser iniciado, em média, após os 9 anos de idade, no período da dentadura mista tardia. Iniciar o tratamento antes dessa época prolonga demasiadamente o tempo de tratamento ${ }^{84}$. As considerações que seguem são aplicáveis em más oclusões de Classe II com arco inferior sem apinhamento ou com um grau de apinhamento passivel de correção sem extrações inferiores. Se o principal responsável pela má oclusão é a protrusão dos dentes superiores, associado ou não à protrusão maxilar, o melhor protocolo é a utilização de um aparelho extrabucal para a distalização dos dentes superiores e/ou restrição do crescimento maxilar para anterior ${ }^{22}$. Se o caso apresentar protrusão dos dentes superiores, associada ou não à protrusão maxilar, e retrusão dos dentes inferiores, associada ou não à retrusão mandibular, o melhor seria combinar a força extrabucal com aparelhos funcionais e/ou elásticos de Classe II, que atuarão nos elementos que estão mal posicionados ${ }^{43}$. Se o problema for exclusivamente devido à uma retru- são dos dentes inferiores, associada ou não a uma retrusão mandibular, o melhor protocolo consistiria na utilização de aparelhos funcionais, fixos ou removíveis $^{32,66}$. Como a resposta das alterações dentoaolveolares e esquelética é mais lenta com esses aparelhos $^{11,12}$, é interessante também se associar a força extrabucal e/ou elásticos de Classe II por um período limitado de tempo diário, para aumentar a eficiência do tratamento ${ }^{43,71}$. À medida que a idade aumenta, menor a probabilidade de correção da discrepância ântero-posterior, sem extrações. De tal forma que, dos 12 anos para frente, em uma Classe II completa, é preferível se corrigir o problema com extrações de 2 pré-molares superiores. Severidades menores de discrepância ântero-posterior podem apresentar maior proporção de sucesso após essa idade. Quando o apinhamento inferior for acentuado, associado ou não à protrusão labial, o caso terá que ser tratado com extrações de quatro prémolares. Nessa situação, o paciente deverá ser extremamente colaborador, se a discrepância ânteroposterior for acentuada. Caso contrário, o resultado final de correção da relação ântero-posterior será comprometido ${ }^{39}$.

\section{7) Da mesma forma, qual seria o melhor tratamento para a má oclusão de Classe II para pacientes não colaboradores, tendo em vista a eficácia do tratamento? Nelson Mucha}

Complementando as considerações anteriores, para casos sem apinhamento inferior ou com apinhamento passivel de correção sem extrações inferiores, se o paciente não é colaborador, a opção será tratá-lo com extrações de dois pré-molares superiores. Se a Classe II for completa, ainda haverá necessidade de colaboração do paciente em utilizar o aparelho extrabucal para manter a ancoragem. $\mathrm{O}$ uso de uma barra palatina diminui, mas não elimina, a necessidade do aparelho extrabucal, nessa situação. Se realmente o paciente não utiliza, de forma nenhuma, o aparelho extrabucal, o jeito é recorrer aos mini-implantes, que proporcionarão uma ancoragem efetiva e absoluta ${ }^{53,70}$. 
18) Tomei conhecimento esta semana, de acordo com o Jornal do SCDRJ, que no Brasil ocorreu um aumento de 154 cursos de Especialização em Ortodontia em 2004, para 227 cursos reconhecidos e credenciados em 2005, correspondendo a um aumento do número de cursos de $47,40 \%$, em apenas um ano. Qual a sua opinião a respeito e quais as soluções apontadas para melhorar a situação da Ortodontia brasileira? Nelson Mucha

Evidentemente esse crescimento acentuado e desordenado não é bom para a especialidade. Com um aumento dessa magnitude é muito provável que haja uma perda da qualidade do ensino e que o nível da Ortodontia brasileira se abaixe ao invés de se elevar. As soluções que poderiam melhorar a situação consistem em se estabelecer critérios mínimos a serem seguidos para que os cursos possam ser credenciados e que sejam vistoriados para que se cumpram os requisitos. Além disso, a abertura de cursos deveria se restringir a áreas em que necessita-se de profissionais e não em áreas já saturadas de especialistas. Como no momento está ocorrendo um fechamento de diversos cursos de graduação de escolas particulares, creio que o reflexo disso será também uma diminuição no credenciamento de novos cursos, uma vez que os mesmos são abertos quando há demanda.

\section{REFERÊNCIAS}

1. ALAVI, D. G.; BEGOLE, E. A.; SCHNEIDER, B. J. Facial and dental arch asymmetries in Class II subdivision malocclusion. Am J Orthod Dentofacial Orthop, St. Louis, v. 93, no. 1, p. 38-46, Jan. 1988.

2. ANDREWS, L. F. The straight wire appliance: syllabus of philosophy and techniques. 2nd ed. San Diego: Larry F. Andrews; Foundation of Orthodontic Education and Research, 1975.

3. ANGELOPOULOS, G. Long-term stability of temporomandibular joint remodelling following continuous mandibular advancement in the juvenile macaca fascicularis [microform] a histomorphometric, cephalometric and electromyographic investigation. 1991. $273 \mathrm{f}$. Thesis (Master)-University of Toronto, Toronto, 1991

4. ANGLE, E. H. Treatment of malocclusion of the teeth. Philadelphia: S. S. White, 1907.

5. BARROS, S. E. C. Avaliação do grau de eficiência do tratamento da Classe II realizado sem extrações e com extrações de dois pré-molares superiores. 2004. $152 \mathrm{f}$. Dissertação (Mestrado)-Faculdade de Odontologia de Bauru, Universidade de São Paulo, Bauru, 2004.
6. BISHARA, S. E.; CUMMINS, D. M.; JAKOBSEN, J. R. The morphologic basis for the extraction decision in Class II, division 1 malocclusions: a comparative study. Am J Orthod Dentofacial Orthop, St. Louis, v. 107, no. 2, p. 129-35, Feb. 1995.

7. BISHARA, S. E.; ZIAJA, R. R. Functional appliances: a review. Am J Orthod Dentofacial Orthop, St. Louis, v. 95, no. 3, p. 250-258, Mar. 1989

8. BLECHMAN, A. M. Magnetic force systems in orthodontics: clinical results of a pilot study. Am J Orthod, St. Louis, v. 87, no. 3, p. 201-210, Mar. 1985.

9. BRYK, C.; WHITE, L. W. The geometry of Class II correction with extractions. J Clin Orthod, Boulder, v. 35, no. 9, p. 570-579, Sept. 2001.

10. BURSTONE, C. J.; OIN, B.; MORTON, J. Y. Chinese NiTi wire: a new orthodontic alloy. Am J Orthod, St. Louis, v. 87, no. 6, p. 445-452, June 1985.

11. CANÇADO, R. H. Estudo comparativo dos resultados oclusais e da eficiência dos protocolos de tratamento em uma e duas fases da má oclusão Classe II, Divisão 1. 2005. 176 p. Tese (Doutorado) - Faculdade de Odontologia de Bauru, Universidade de São Paulo.

12. CANÇADO, R. H.; PINZAN, A.; JANSON, G.; HENRIQUES, J. F. C.; NEVES, L. S.; CANUTO, C. E. Occlusal outcomes and efficiency of 1- and 2-phase protocols in Class II, division 1 malocclusion treatment. Am J Orthod Dentofacial Orthop, St. Louis, 2006. In press.

13. CARANO, A.; TESTA, M. The distal jet for upper molar distalization. J Clin Orthod, Boulder, v. 30, no 7, p. 374-380, July 1996.

14. CHEN, J. Y.; WILL, L. A.; NIEDERMAN, R. Analysis of efficacy of functional appliances on mandibular growth. Am J Orthod Dentofacial Orthop, St. Louis, v. 122, no. 5, p. 470-476, Nov. 2002.

15. CHENEY, E. A. The influence of dentofacial asymmetry upon treatment procedures. Am J Orthod, St. Louis, v. 38, no. 2, p. 934-945, July 1952.

16. CHENEY, E. A. Dentofacial asymmetries and their clinical significance. Am J Orthod, St. Louis, v. 47, no. 2, p. 814-829, 1961.

17. COELHO FILHO, C. M. Mandibular protraction appliances for Class II treatment. J Clin Orthod, Boulder, v. 29, no. 5, p. 319-336, May 1995.

18. COELHO FILHO, C. M. Clinical applications of the mandibular protraction appliance. J Clin Orthod, Boulder, v. 31, no. 2, p. 92-102, Feb. 1997.

19. COELHOFILHO, C. M. The mandibular protraction appliance no. 3 . J Clin Orthod, Boulder, v. 32, no. 6, p. 379-384, Jun. 1998.

20. COELHO FILHO, C. M. Mandibular protraction appliance no. 4. J Clin Orthod, Boulder, v. 35, no. 1, p. 18-24, Jan. 2001.

21. COHEN, J. Statistical power analysis for the behavioral sciences. Hillsdale, New Jersey: Erlbaum Associates, 2 ed. 1988.

22. CREEKMORE, T. D. Inhibition or stimulation of the vertical growth of the facial complex, its significance to treatment. Angle Orthod, Appleton, v. 37, no. 4, p. 285-297, Oct. 1967.

23. DAWSON, P. E. Evaluation, diagnosis, and treatment of occlusal problems. 2nd ed. St. Louis: C. V. Mosby, 633p. 1989.

24. ECKHART, J. E. Introducing the MARA. Clin Impress, [S. I.], v. 7, no. 3, p. 2-5, 1998.

25. FORTINI, A.; LUPOLI, M.; PARRI, M. The first class appliance for rapid molar distalization. J Clin Orthod, Boulder, v. 33, no. 6, p. 322-328, June 1999

26. FREITAS, K. M.; DE FREITAS, M. R.; HENRIQUES, J. F. C.; PINZAN, A.; JANSON, G. Postretention relapse of mandibular anterior crowding in patients treated without mandibular premolar extraction. Am J Orthod Dentofacial Orthop, St. Louis, v. 125, no. 4, p. 480-487, Apr. 2004.

27. FREITAS, M. R.; BELTRAO, R. T.; JANSON, G.; HENRIQUES, J. F.; CANCADO, R. H. Long-term stability of anterior open bite extraction treatment in the permanent dentition. Am J Orthod Dentofacial Orthop, St. Louis, v. 125, no. 1, p. 78-87, Jan. 2004.

28. FUZIY, A.; ALMEIDA, R. R.; JANSON, G.; ANGELIERI, F.; PINZAN, A. Sagittal, vertical and transverse changes consequent to maxillary molars distalization with the pendulum appliance. Am J Orthod Dentofacial Orthop, St. Louis, 2006. In Press. 
29. FUZIY, A ; JANSON, G. Alterações tegumentares do tratamento da má oclusão de Classe II divisão 1 sem e com extrações de pré-molares superiores. 2005. Tese (Pós-Doutorado)-Departamento de Ortodontia, Universidade de São Paulo, São Paulo, 2005.

30. GIANELLY, A. A.; BEDNAR, J.; DIETZ, V. S. Japanese NiTi coils used to move molars distally. Am J Orthod Dentofacial Orthop, St. Louis, v. 99, no. 6, p. 564-566, June 1991

31. GOLDIN, B. Labial root torque: effect on the maxilla and incisor root apex. Am J Orthod Dentofacial Orthop, St. Louis, v. 95, no. 3, p. 208-219, Mar. 1989

32. GRABER, T. M.; NEUMANN, B. Removable orthodontic appliances. 2nd ed. Philadelphia: Saunders, 631p. 1984

33. GREENE, W. H. Econometric analysis. 4th ed. New Jersey: Prentice-Hall, 2000

34. HILGERS, J. J. The pendulum appliance for Class II non-compliance therapy. J Clin Orthod, Boulder, v. 26, no. 11, p. 706-714, Nov. 1992.

35. JACOBS, T.: SAWAENGKIT, P. National Institute of Dental and Craniofacial Research efficacy trials of bionator Class II treatment: a review. Angle Orthod, Appleton, v. 72, no. 6, p. 571-575, Dec. 2002.

36. JANSON, G. Estudo tridimensional das assimetrias dentárias e esqueléticas na má oclusão de Classe II, subdivisão. 1998. 271 f. Tese (Livre-Docência)-Faculdade de Odontologia de Bauru, Universidade de São Paulo, Bauru, 1998.

37. JANSON, G. Influência do padrão facial no tratamento ortodôntico da Classe III. In: SAKAI, E.; MARTINS, N. S. et al. (Ed.). Nova visão em Ortodontia-Ortopedia Facial. São Paulo: Ed. Santos, 2000. p. 371-378.

38. JANSON, G.; BARROS, S. E. C.; FREITAS, M. R.; HENRIQUES, J. F. C.; PINZAN, A. Class II treatment efficiency in maxillary premolar extraction and non-extraction protocols. Am J Orthod Dentofacial Orthop, St. Louis. In Press.

39. JANSON, G.; BRAMBILLA, A. C.; HENRIQUES, J. F. C.; DE FREITAS, M. R.; NEVES, L. S. Class II treatment success rate in 2- and 4-premolar extraction protocols. Am J Orthod Dentofacial Orthop, St. Louis, v. 125, no. 4, p. 472-479, Apr. 2004.

40. JANSON, G.; CAFFER, D C.; HENRIOUES, J. F. C : DE FREITAS, M. R.; NEVES, L. S. Stability of Class II, division 1 treatment with the headgear-activator combination followed by the edgewise appliance. Angle Orthod, Appleton, v. 74, no. 5, p. 594-604, Oct. 2004.

41. JANSON, G.; CARVALHO, P. E. G.; HENRIQUES, J. F. C.; FREITAS, M. R.; PINZAN, A. Alterações dento-esqueléticas na correção da má oclusão de Classe II subdivisão, com elásticos intermaxilares. Rev Clín Ortodon Dental Press, Maringá, v. 3, n. 2, p. 63-70, abr./maio 004

42. JANSON, G.; DE SOUZA, J. E.; ALVES, F. A.; ANDRADE JR., P.; NAKAMURA, A.; DE FREITAS, M. R.; HENRIQUES, J. F. C. Extreme dentoalveolar compensation in the treatment of Class III malocclusion. Am J Orthod Dentofacial Orthop, St. Louis, v. 128 , no. 6, p. 787-94, Dec. 2005

43. JANSON, G.; HENRIQUES, J. F. C.; FREITAS, M. R. Aparelho ativador associado à força extrabucal. In: BATISTA, J. (Ed.). Livro digital: Ortopedia Facial e Ortodontia. Curitiba: Editek, 2000.

44. JANSON, G.; LIMA, K.; WOODSIDE, D.; METAXAS, A.; FREITAS, M.; HENRIQUES, J. Distribution of Class II subdivision malocclusion types and evaluation of their asymmetries. Am J Orthod Dentofacial Orthop, St. Louis. In Press.

45. JANSON, G.; MARTINS, D. R.; HENRIQUES, J. F. C.; FREITAS, M. R.; PINZAN, A.; ALMEIDA, R. R. Functional occlusion and occlusal adjustment. In: VIAZIS, A. D. (Ed.). Atlas of advanced Orthodontics: a guide to clinical efficiency. Philadephia: Saunders, 1998. p. 203-214

46. JANSON, G.; PUTRICK, L. M.; HENRIQUES, J. F. C.; FREITAS, M. R.; HENRIQUES, R. P. Maxillary third molar position in Class II malocclusions: the effect of treatment with and without maxillary premolar extractions. Eur J Orthod, Oxford, 2006. In Press.

47. JANSON, G.; TORUÑO, J. A.; CRUZ, K. S.; HENRIQUES, J. F. C.; FREITAS, M. R. Tratamento e estabilidade da má oclusão de Classe III. Rev Dental Press Ortodon Ortop Facial, Maringá, v. 7, n. 3, p. 85-94, maio/jun. 2002
48. JANSON, G.; VALARELLI, F. P.; BELTRAO, R. T.; FREITAS, M. R. HENRIQUES, J. F. C. Stability of anterior open-bite extraction and nonextraction treatment in the permanent dentition. Am J Orthod Dentofacial Orthop, St. Louis, v. 129, no. 6, p. 768-774, June 2006

49. JANSON, G.; VALARELLI, F. P.; HENRIQUES, J. F. C.; DE FREITAS M. R.; CANCADO, R. H. Stability of anterior open bite nonextraction treatment in the permanent dentition. Am J Orthod Dentofacial Orthop, St. Louis, v. 124, no. 3, p. 265-276, Sept. 2003.

50. JANSON, G.; WOODSIDE, D. G.; METAXAS, A.; HENRIQUES, J. F. C.; FREITAS, M. R. Orthodontic treatment of subdivision cases. World J Orthod, Carol Stream, v. 4, no. 1, p. 36-46, Springer 2003

51. JANSON, G. R.; METAXAS, A.; WOODSIDE, D. G.; DE FREITAS, M. R.; PINZAN, A. Three-dimensional evaluation of skeletal and dental asymmetries in Class II subdivision malocclusions. Am J Orthod Dentofacial Orthop, St. Louis, v. 119, no. 4, p. 406-418, Apr. 2001.

52. JONES, R. D.; WHITE, J. M. Rapid Class II molar correction with an open-coil jig. J Clin Orthod, Boulder, v. 26, no. 10, p. 661-664 Oct. 1992.

53. KANOMI, R. Mini-implant for orthodontic anchorage. J Clin Orthod, Boulder, v. 31, no. 11, p. 763-767, Nov. 1997.

54. KEELING, S. D. et al. Anteroposterior skeletal and dental changes after early Class II treatment with bionators and headgear. Am J Orthod Dentofacial Orthop, St. Louis, v. 113, no. 1, p. 40-50, Jan. 1998.

55. KIM, T. W.; ARTUN, J.; BEHBEHANI, F.; ARTESE, F. Prevalence of third molar impaction in orthodontic patients treated nonextraction and with extraction of 4 premolars. Am J Orthod Dentofacial Orthop, St. Louis, v. 123, no. 2, p. 138-145, Feb. 2003

56. KLAPPER, L. The Super spring II: a new appliance for non-compliant Class II patients. J Clin Orthod, Boulder, v. 33, no. 1, p. 50-54, Jan. 1999.

57. LIMA, K. J. R. S. Distribuição dos tipos de Classe II, subdivisão e avaliação de suas assimetrias. 2003. 176 f. Dissertação (Mestrado) - Faculdade de Odontologia de Bauru, Universidade de São Paulo.

58. LITTLE, R. M.; RIEDEL, R. A. Postretention evaluation of stability and relapse--mandibular arches with generalized spacing. Am J Orthod Dentofacial Orthop, St. Louis, v. 95, no. 1, p. 37-41, Jan. 1989

59. LITTLE, R. M.; RIEDEL, R. A.; ARTUN, J. An evaluation of changes in mandibular anterior alignment from 10 to 20 years postretention. Am J Orthod Dentofacial Orthop, St. Louis, 93, no. 5 p. 423-428, May 1988.

60. LITTLE, R. M.; WALLEN, T. R.; RIEDEL, R. A. Stability and relapse of mandibular anterior alignment-first premolar extraction cases treated by traditional edgewise orthodontics. Am J Orthod, St. Louis, v. 80, no. 4, p. 349-365, Oct. 1981

61. MAILANKODY, J. Enigma of Class II molar finishing. Am J Orthod Dentofacial Orthop, St. Louis, v. 126, no. 6, p. A15-6; author reply A16-7, Dec. 2004.

62. ARAUjO, T. M.; WILHELM, R. S.; ALMEIDA, M. A. Skeletal and dental arch asymmetries in Class II division 1 subdivision malocclusions. J Clin Pediatr Dent, Birmingham, v. 18, no. 3 p. 181-185, Spring. 1994

63. METAXAS, A. Primate experiments in bone remodeling in the temporomandibular joint and facial complex using the Herbst appliance. 1983. Master's (Thesis)-Department of Orthodontics, University of Toronto, Toronto, 1983.

64. MIURA, F.; MOGI, M.; OHURA, Y.; HAMANAKA, H. The superelastic property of the Japanese $\mathrm{NiTi}$ alloy wire for use in orthodontics. Am J Orthod Dentofacial Orthop, St. Louis, v. 90 no. 1, p. 1-10, July 1986

65. O'BRIEN, K. D.; ROBBINS, R.; VIG, K. W.; VIG, P. S.; SHNORHOKIAN, H.; WEYANT, R. The effectiveness of Class II, division 1 treatment. Am J Orthod Dentofacial Orthop, St. Louis, v. 107, no. 3 p. 329-334, Mar. 1995.

66. PANCHERZ, H. Treatment of Class II malocclusions by jumping the bite with the Herbst appliance: a cephalometric investigation. Am J Orthod, St. Louis, v. 76, no. 4, p. 423-442, Oct. 1979. 
67. PANCHERZ, $\mathrm{H}$. The nature of Class II relapse after Herbst appliance treatment: a cephalometric long-term investigation. Am J Orthod Dentofacial Orthop, St. Louis, v. 100, no. 3, p. 220-333, Sept. 1991.

68. PANCHERZ, H.; ANEHUS-PANCHERZ, M. The headgear effect of the Herbst appliance: a cephalometric long-term study. Am J Orthod Dentofacial Orthop, St. Louis, v. 103, no. 6, p. 510-520, June 1993.

69. PANDIS, N.; POLYCHRONOPOULOU, A.; ELIADES, T. Failure rate of self-ligating and edgewise brackets bonded with conventional acid etching and a self-etching primer: a prospective in vivo study. Angle Orthod, Appleton, v. 76, no. 1, p. 119-122, Jan. 2006.

70. PARK, H. S.; BAE, S. M.; KYUNG, H. M.; SUNG, J. H. Microimplant anchorage for treatment of skeletal Class I bialveolar protrusion. J Clin Orthod, Boulder, v. 35, no. 7, p. 417-422, July 2001.

71. PFEIFFER, J. P.; GROBETY, D. Simultaneous use of cervical appliance and activator: an orthopedic approach to fixed appliance therapy. Am J Orthod, St. Louis, v. 61, no. 4, p. 353-373, Apr. 1972.

72. PINZAN-VERCELINO, C. R. M. Comparação entre os graus de eficiência no tratamento da má oclusão de Classe II realizado com o aparelho pendulum e com as extrações de dois prémolares superiores. 2005. $158 \mathrm{f}$. Tese (Doutorado)-Faculdade de Odontologia de Bauru, Universidade de São Paulo, Bauru, 2005.

73. PROFFIT, W. R. Contemporary treatment of dentofacial deformity. St. Louis: C. V. Mosby, 751p. 2003.

74. PUTRICK, L. M. Presença clínica e angulação dos terceiros molares superiores, na má oclusão de Classe II, tratada sem e com extrações de pré-molares superiores. 2003. 74 f. Dissertação (Mestrado)-Departamento de Ortodontia, Faculdade de odontologia de Bauru, Universidade de São Paulo, São Paulo, 2003

75. ROCK, W. P. Treatment of Class II malocclusions with removable appliances. Part 4. Class II division 2 treatment. Br Dent J, London, v. 168, no. 7, p. 298-302, Apr. 1990

76. ROSE, J. M.; SADOWSKY, C.; BEGOLE, E. A.; MOLES, R. Mandibular skeletal and dental asymmetry in Class II subdivision malocclusions. Am J Orthod Dentofacial Orthop, St. Louis, v. 105, no. 5, p. 489-495, May 1994.

77. ROTH, R. H. Functional occlusion for the orthodontist. J Clin Orthod, Boulder, v. 15, no. 1, p. 32-40, 40-51, Jan. 1981.

78. SARVER, D. M. New concepts in esthetics and appearance, and the new trends in orthodontic treatment planning. Journalbytes, [S.I.], v. 12, no. 12, 2001. Disponível em: <http://www.journalbytes.com>. Acesso em: 12 jan. 2006.

79. SHEATS, R. D.; PANKRATZ, V. S. Common statistical tests. Semin Orthod, Philadelphia, v. 8, no. 2, p. 77-86, June 2002.

80. SHIELDS, T. E.; LITTLE, R. M.; CHAPKO, M. K. Stability and relapse of mandibular anterior alignment: a cephalometric appraisal of first-premolar-extraction cases treated by traditional edgewise orthodontics. Am J Orthod, St. Louis, v. 87, no. 1, p. 27-38, Jan. 1985.

81. SIQUEIRA, D. F.; ALMEIDA, R. R.; JANSON, G.; BRANDÃO, A. G.; COELHO FILHO, C. M. Dento skeletal and soft tissue changes of cervical headgear and mandibular protraction appliance in Class II malocclusion treatment. Am J Orthod Dentofacial Orthop, St. Louis, 2006. In press.

82. STEINER, C. C. Cephalometrics as a clinical tool. In: KRAUS, B. S. RIEDEL, R. A. (Ed.). Vistas in Orthodontics. Philadelphia: Lea \& Febiger, 1962. p. 131-161.

83. TWEED, C. H. Indications for the extraction of teeth in orthodontic procedure. Am J Orthod, St. Louis, v. 30, no. 8, p. 405-428, Aug. 1944.

84. VON BREMEN, J.; PANCHERZ, H. Efficiency of early and late Class II Division 1 treatment. Am J Orthod Dentofacial Orthop St. Louis, v. 121, no. 1, p. 31-37, Jan. 2002

85. VOUDOURIS, J. C.; KUFTINEC, M. M. Improved clinical use of Twin-block and Herbst as a result of radiating viscoelastic tissue forces on the condyle and fossa in treatment and long-term retention: growth relativity. Am J Orthod Dentofacial Orthop, St. Louis, v. 117, no. 3, p. 247-266, Mar. 2000.
86. VOUDOURIS, J. C. et al. Condyle-fossa modifications and muscle interactions during Herbst treatment. Part 2. Results and conclusions. Am J Orthod Dentofacial Orthop, St. Louis, v. 124, no. 1, p. 13-29, July 2003

87. VOUDOURIS, J. C.; WOODSIDE, D. G.; ALTUNA, G.; KUFTINEC, M. M.; ANGELOPOULOS, G.; BOURQUE, P. J. Condyle-fossa modifications and muscle interactions during Herbst treatment, part 1. New technological methods. Am J Orthod Dentofacial Orthop, St. Louis, v. 123, no. 6, p. 604-613, June 2003.

88. WAINWRIGHT, W. Faciolingual tooth movement: its influence on the root and cortical plate. Am J Orthod, St. Louis, v. 64, no. 3, p. 278-302, Sept. 1973

89. WERTZ, R. A. Diagnosis and treatment planning of unilateral Class II malocclusions. Angle Orthod, Appleton, v. 45, no. 2, p. 85-94, Apr. 1975.

90. WEST, R. P. The adjustable bite corrector. J Clin Orthod, Boulder, v. 29 , no. 10 , p. $650-657$, Oct. 1995.

91. WOODSIDE, D. G.; METAXAS, A.; ALTUNA, G. The influence of functional appliance therapy on glenoid fossa remodeling. Am JOrthod Dentofacial Orthop, St. Louis, v. 92, no. 3, p. 181-198, Sept. 1987.

92. WYLIE, G. A.; FISHER, L. C.; EPKER, B. N. Cephalometrics: a comparison of five analyses currently used in the diagnosis of dentofacial deformities. Int J Adult Orthodon Orthognath Surg, Chicago, v. 2, no. 1, p. 15-36, 1987

93. ZACHRISSON, B. U. Use of self-ligating brackets, superelastic wires, expansion/proclination, and permanent retention: a word of caution. World J Orthod, Carol Stream, v. 7, no. 2, p. 198-206, Summer 2006

Francisco Antonio Bertoz

- Professor Titular do Departamento de Odontologia Infantil e Social da Faculdade de Odontologia do Campus de Araçatuba-UNESP;

- Professor colaborador da Disciplina de Ortodontia da Faculdade de Odontologia do Campus de Araçatuba-UNESP. - Coordenador da Área de Ortodontia do Programa Pós-Graduação em Odontologia da FOA/UNESP (Stricto Sensu); - Coordenador do Curso de Pós-Graduação em Ortodontia (Lato Sensu).

Maria Fernanda Martins-Ortiz Consolaro

- Mestre em Ortodontia pela FOB - USP;

- Doutora em Patologia pela FOB - USP;

- Clínica Privada em Bauru - SP.

\section{Nelson Mucha}

Graduado pela Faculdade de Odontologia da Universidade de Passo Fundo - RS;

- Especialista em Prótese Dental pela Faculdade de Odontologia de Piracicaba - Unicamp - SP.

- Especialista em Radiologia pela SBO-UFRJ;

- Mestre em Ciências - Ortodontia - UFRJ;

- Doutor em Odontologia - Ortodontia - UFRJ;

- Professor Titular de Ortodontia da Universidade Federa

Fluminense - UFF - Niterói - RJ;

- Diretor do Board Brasileiro de Ortodontia - BBO.

Osmar Aparecido Cuoghi

- Professor Doutor e Chefe do Departamento de Odontologia Infantil e Social - Disciplina de Ortodontia - UNESP/Araçatuba;

- Professor do Programa de Pós-Graduação em Odontologia; - Área de Ortodontia - UNESP/Araçatuba. 